\title{
Caregiver Burden in Alzheimer's Disease: Differential Associations in Adult-Child and Spousal Caregivers in the GERAS Observational Study
}

\author{
Catherine Reed ${ }^{\mathrm{a}} \quad$ Mark Belger $^{\mathrm{a}} \quad$ Grazia Dell'Agnello $^{\mathrm{c}}$ Anders Wimo $^{\mathrm{e}}$ \\ Josep Maria Argimon ${ }^{f} \quad$ Giuseppe Bruno $^{d}$ Richard Dodel ${ }^{\text {h }}$ \\ Josep Maria Harog ${ }^{g}$ Roy W. Jones ${ }^{b}$ Bruno Vellas ${ }^{i}$
}

${ }^{a}$ Eli Lilly and Company Limited, Windlesham, and ${ }^{b}$ RICE (The Research Institute for the Care of Older People), Bath, UK; ' Lilly Italy, Florence, and 'University of Rome 'Sapienza', Rome,

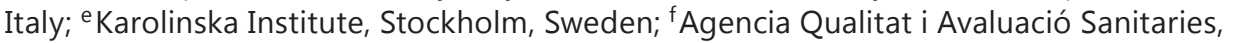
and 9 Parc Santari Sant Joan de Déu, CIBERSAM, Universitat de Barcelona, Barcelona, Spain; hPhilipps University, Marburg, Germany; 'Toulouse University Hospital, INSERM 1027, Toulouse, France

Key Words

Adult-child caregiver - Alzheimer's disease - Europe · Observational study · Spousal caregiver

\section{Abstract}

Background/Aims: To examine factors influencing the caregiver burden in adult-child and spousal caregivers of community-dwelling patients with Alzheimer's disease (AD). Methods: Baseline data from the 18-month, prospective, observational GERAS study of 1,497 patients with AD in France, Germany, and the UK were used. Analyses were performed on two groups of caregivers: spouses $(n=985)$ and adult children $(n=405)$. General linear models estimated patient and caregiver factors associated with subjective caregiver burden assessed using the Zarit Burden Interview. Results: The caregiver burden increased with AD severity. Adult-child caregivers experienced a higher burden than spousal caregivers despite spending less time caring. Worse patient functional ability and more caregiver distress were independently associated with a greater burden in both adult-child and spousal caregivers. Additional factors were differentially associated with a greater caregiver burden in both groups. In adult-child caregivers these were: living with the patient, patient living in an urban location, and patient with a fall in the past 3 months; in spouses the factors were: caregiver gender (female) and age (younger), and more years of patient education. Conclusion: The perceived burden differed between adult-child and spousal caregivers, and specific patient and caregiver factors were differentially associated with this burden. 
Reed et al.: Caregiver Burden in Alzheimer's Disease: Differential Associations in Adult-Child and Spousal Caregivers in the GERAS Observational Study

\section{Introduction}

Alzheimer's disease (AD), the most common cause of dementia, presents an enormous health, social, economic, and personal challenge given the large and growing number of older people affected by the disease worldwide [1-3]. Informal caregivers, typically family members, play a major role in caring for $\mathrm{AD}$ patients living at home, and the cost of informal care in $\mathrm{AD}$ represents the largest component of societal costs [4,5]. Caregiver burden can be defined as the caregiver's perception of the physical, emotional, economic, and social cost of the caregiving relationship [6]. Identifying patient and caregiver characteristics associated with the caregiver burden is an important step towards determining interventions that may alleviate the burden of caring for patients with $\mathrm{AD}$ and may result in cost savings [7].

Increased caregiver burden has been associated with increased risk of AD patient hospitalization [8] and a faster time to institutionalization and death in AD patients [8-10], as well as early mortality for caregivers themselves [11]. This is despite the positive aspects of caregiving, which include companionship, reward, and enjoyment $[6,12]$.

The caregiver burden increases with greater AD severity, and both patient and caregiver characteristics have been found to explain greater caregiver burden, with some differences depending on the caregiver-patient relationship $[6,13,14]$. Although the caregiver relationship to the patient with $\mathrm{AD}$ is often reported amongst the potential factors affecting caregiver burden, findings of different studies are conflicting. In some studies, adult children experienced the highest level of burden [15-18], in others spouses had a higher burden [1923], whereas some other studies reported no significant differences in burden between these caregiver groups [24-26] or that the caregiver relationship to the patient was not a determinant of caregiver burden [27-29]. Conde-Sala et al. [13] directly compared factors associated with burden in caregivers who were adult children or spouses of AD patients. Using the Zarit Burden Interview (ZBI), a widely used instrument for measuring subjective caregiver burden in $\mathrm{AD}$ [30], a greater level of burden was found in adult-child caregivers than in spousal caregivers [13]. This highlights the caregiver relationship to the patient as an important factor when assessing caregiver burden. However, further studies are required as the Conde-Sala study was conducted in an individual country (Spain) and included relatively small samples of adult-child and spousal/partner caregivers [13]. Differences in the burden perceived by spousal and adult-child caregivers may also be influenced by disease severity $[13,31]$. Previous studies of community-dwelling AD patients focused mainly on patients with milder forms of the disease or did not directly report burden according to AD severity, but we have recently found in the GERAS study, a large multinational observational study that included a relatively large number of patients with more severe $\mathrm{AD}$, that caregiver burden increased with increasing AD severity [32]. Exploring factors influencing caregiver burden separately in adult-child and spousal caregivers of a large population of patients with mild, moderate and more severe AD will identify differential factors and allow better targeting or tailoring of interventions to reduce caregiver burden.

The objectives of this study were to explore the associations between patient and caregiver characteristics and clinical factors, and subjective caregiver burden in adult-child and spousal caregivers participating in the GERAS study.

\section{Methods}

\section{Study Design and Participants}

GERAS is an 18-month prospective, multicenter, naturalistic, observational cohort study reflecting the routine care of patients with AD in France, Germany, and the UK. The main aims 
of the study were to evaluate costs and resource use associated with AD for patients and caregivers. The study design and methods have been described in detail elsewhere [4].

Briefly, investigators, mostly from specialist secondary care clinics ('memory clinics'), enrolled community-dwelling patients aged at least 55 years, diagnosed with probable AD according to the National Institute of Neurological and Communicative Disorders and Stroke and Alzheimer's Disease and Related Disorders Association (NINCDS-ADRDA) criteria [33], with a Mini-Mental State Examination (MMSE) [34] score of $\leq 26$, and who presented within the normal course of care. Patients with other potential causes of dementia were excluded from the study. Enrolled patients were also required to have a primary caregiver who was willing to participate in the study and be responsible for the patient for at least 6 months of the year. 'Primary caregiver' was defined as an informal carer who, according to the family, was the person who normally takes most responsibility for the day-to-day decisions and provision of home care for the patient.

All patients (or their legal representative) and caregivers were required to provide written informed consent before entering the study, which was approved by ethics review boards in each country according to country-specific regulations. Study enrolment was from October 2010 to September 2011. Patients and caregivers were evaluated at baseline and could attend up to three assessment visits at 6-month intervals during the 18-month study period, thus reflecting the routine care of patients with AD. This article reports a post hoc analysis of the baseline data from this study.

Patients were stratified according to disease severity at baseline using MMSE criteria based on UK clinical guidelines [35]: mild AD (MMSE score = 21-26), moderate AD (MMSE score $=15-20$ ) or moderately severe/severe AD (MMSE score $<15$ ). As the study aimed to recruit equal numbers of patients in each of the three severity groups, the sample size was calculated on this basis together with a potential dropout rate of $20 \%$ each year during the study, as reported previously [4].

\section{Data Collected}

Patient information collected at baseline included age, gender, marital status, number of years of education, time since AD diagnosis, living arrangements, comorbidities and whether or not they had experienced a fall in the past 3 months. Cognitive function was tested using the MMSE [34] and, for patients with mild or moderate disease severity, the cognitive subscale of the Alzheimer's Disease Assessment Scale [36]; these scales were completed by the investigators. Patient functional ability was assessed by caregivers using the Alzheimer's Disease Co-operative Study of Activities of Daily Living Inventory (ADCS-ADL) [37]. Behavioral symptoms, and caregiver distress caused by these symptoms, were evaluated by caregivers using the Neuropsychiatric Inventory (NPI) [38, 39].

Caregiver information collected at baseline included age, gender, relationship to the patient, marital status, whether they live with the patient (yes/no), whether they are the sole caregiver (yes/no), employment status (working/not working for pay), and medical conditions.

Caregiver burden was assessed using the 22-item ZBI [30], a self-reported instrument that includes questions on caregiver stress, time available for self, and impact of caring on the caregivers' social life. Responses to each item are recorded on a 5-point scale $(0=$ never, $4=$ nearly always). The ZBI total score has a range of $0-88$, with higher scores indicating greater burden.

Caregivers completed both the caregiver and patient-proxy versions of the EuroQol-5 Dimension Questionnaire (EQ-5D) [40], providing estimates of both the caregiver's and patient's health-related quality of life (HRQoL). 
Reed et al.: Caregiver Burden in Alzheimer's Disease: Differential Associations in Adult-Child and Spousal Caregivers in the GERAS Observational Study

Caregiver resource use during the month before the baseline visit was assessed using the Resource Utilization in Dementia (RUD) instrument [41]. The RUD was administered by the physician and answered by the caregiver. This included information on the time spent caring for the patient in three distinct areas of care: the number of hours spent on assisting basic activities of daily living (ADL), the number of hours spent on instrumental ADL, and supervision time.

\section{Statistical Analysis}

Analyses were performed on two groups of caregivers: adult children and spouses. Caregivers whose relationship to the patient was reported as friend or any other relationship were excluded from this analysis. Relationship status was collected using the RUD questionnaire, and the responses for husbands and wives were combined to form the spousal group (which may have included the patient's partner in some cases as the RUD does not specify how people who are partners or cohabiting with the patient should respond).

Descriptive statistics (means and SDs or frequencies) were used to summarize all variables, including demographic and clinical characteristics, and were based on nonmissing observations. Comparisons between caregiver groups used Cochran-Mantel-Haenszel tests for categorical data, stratified by country and MMSE severity group. For continuous variables, the $\mathrm{p}$ values were taken from the type III sum of squares general linear regression model (using PROC GLM in SAS), which assumes the data are normally distributed, and includes the factors caregiver-patient relationship, MMSE severity group and country.

A backward selection method was used for separate multivariate general linear models (GLMs, using PROC GENMOD with the identity link function) for the adult-child and the spouse cohorts to identify which patient and caregiver factors were independently associated with the ZBI total score. This model assumes that the probability distribution is normal. Country and MMSE severity group were forced into the model while other factors were removed from the model based on an exclusion criterion of $\mathrm{p}>0.05$. In addition to country and MMSE severity group, the variables included in the initial model prior to the backward selection process were: (1) patient factors: age, gender, living location (urban/rural), ADCS-ADL total score, years of education, duration of disease, experienced a fall in the past 3 months, and number of comorbidities, and (2) caregiver factors: age, gender, lives with patient (yes/no), sole caregiver (yes/no), working for pay (yes/no), number of medical conditions, NPI distress score, time spent caring for basic ADL, instrumental ADL, and supervision time. The deviance $R^{2}\left(R^{2}\right.$ DEV $)$ was calculated for each of the GLMs.

Sensitivity analyses were used to explore the relationship between cognitive and functional measures on the ZBI. The ADCS-ADL score was removed from the model selection, while keeping the MMSE score in the model. For functionality, the relationship between the ADCS-ADL and ZBI was explored in both caregiver groups through alternative GLMs which replaced the total ADL score with the ADL-instrumental and ADL-basic subdomain scores, and with the scores for the four subdomains of basic activities, household activities, communication, and outdoor activities.

Because caregivers of patients with dementia report high rates of depression [10,42] and depression has been reported as a predictor of caregiver burden as assessed using the ZBI [43], we wanted to investigate the impact of caregiver depression on burden in our study population. A further GLM included the presence of caregiver depression (yes/no) in the final model. All sensitivity analyses for the adult-child model were run with the 'caregiver lives with the patient' factor included. The different sensitivity analyses were compared using the Akaike information criterion (AIC) model fit statistic from the GLMs.

All data were analyzed using SAS software, version 9.2 (SAS Institute, Cary, N.C., USA). Statistical significance was considered to be $\mathrm{p}<0.05$. 
Reed et al.: Caregiver Burden in Alzheimer's Disease: Differential Associations in Adult-Child and Spousal Caregivers in the GERAS Observational Study

Fig. 1. Mean ZBI total scores by patient $\mathrm{AD}$ severity group for adult-child and spousal caregivers. Data presented are unadjusted mean ZBI scores. Error bars indicate 95\% confidence intervals. Mild AD = MMSE score 21-26; moderate AD = MMSE score 1520 , and moderately severe/severe $\mathrm{AD}=$ MMSE score $<15$.

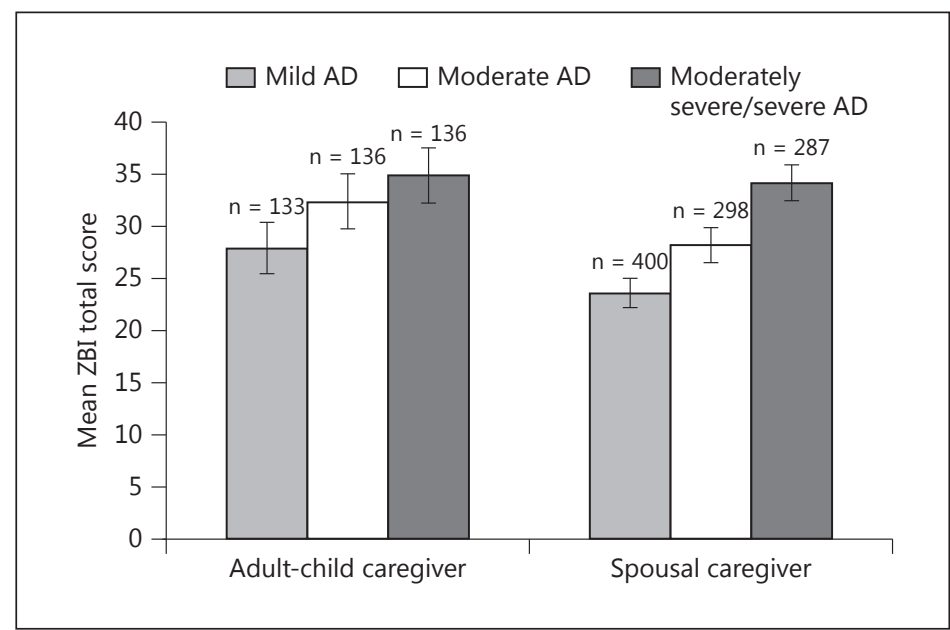

\section{Results}

The study cohort included 1,497 patients with AD, 985 with spousal caregivers and 405 with adult-child caregivers. The remaining 107 patients were excluded from the analysis because their primary caregiver at baseline was reported as being a friend or another person who was not the spouse or adult child of the patient (caregiver-patient relationship status was missing for 3 patients). Thus, a total of 1,390 caregivers were analyzed: 985 (70.9\%) spousal caregivers and 405 (29.1\%) adult-child caregivers.

Table 1 summarizes the patient and caregiver characteristics at baseline by caregiver relationship to the patient. Patients with adult-child caregivers were older, predominantly women (82.5\%), mostly widowed (75.8\%), had a shorter mean time since AD diagnosis and a shorter duration of education, $49.1 \%$ lived alone in their own home, and $18.9 \%$ had experienced a fall in the past 3 months. The adult-child caregivers were mostly women $(74.6 \%)$, $58.3 \%$ were working for pay, and $27.2 \%$ were the sole caregiver, whereas the spousal caregivers were older, fewer were women (58.8\%), $72.0 \%$ were the sole caregiver, $91.5 \%$ were not working for pay, and they had a higher number of medical conditions. Almost all (99.2\%) of the spousal caregivers lived with the patient.

In the adult-child caregiver group, there were 133 (32.8\%), 136 (33.6\%) and $136(33.6 \%)$ patients with mild, moderate and moderately severe/severe AD, respectively; the corresponding numbers in the spousal caregiver group were 400 (40.6\%), 298 (30.3\%) and 287 (29.1\%). The caregiver burden (unadjusted mean ZBI total score) was higher with greater AD severity in both groups of caregivers (fig. 1). A GLM of the ZBI using the MMSE severity group, caregiver-patient relationship and the interaction between the two as factors showed that both the MMSE severity group ( $p<0.001$; i.e., greater burden with higher AD severity) and caregiver-patient relationship ( $p<0.001$; i.e., adult-child caregivers have a higher burden than spousal caregivers) were statistically significantly associated with ZBI total score, but that the interaction between MMSE severity and caregiver-patient relationship was not significant $(p=0.23)$. Thus, there is no evidence that ZBI total score behaves differently across AD severity groups depending on the type of caregiver.

Table 2 reports the least square mean differences and associated $p$ values for the caregiver-patient relationship factor in the GLMs for baseline clinical characteristics. The models show some differences in clinical characteristics between patients cared for by adult-child caregivers versus spousal caregivers. Although the patients with adult-child caregivers had a 
Table 1. Characteristics of patients and caregivers at baseline by caregiver relationship to the patient

\begin{tabular}{|c|c|c|c|}
\hline Characteristic & $\begin{array}{l}\text { Adult-child caregiver } \\
(\mathrm{n}=405)\end{array}$ & $\begin{array}{l}\text { Spousal caregiver } \\
(\mathrm{n}=985)\end{array}$ & $\mathrm{p}$ value \\
\hline \multicolumn{4}{|l|}{ Patients } \\
\hline Age, years & $81.5 \pm 6.6$ & $75.9 \pm 7.5$ & $<0.001$ \\
\hline Female gender & $334(82.5 \%)$ & $410(41.6 \%)$ & $<0.001$ \\
\hline \multicolumn{4}{|l|}{ Marital status ${ }^{b}$} \\
\hline Married & $69(17.0 \%)$ & - & \\
\hline Widowed & $307(75.8 \%)$ & - & \\
\hline Divorced & $26(6.4 \%)$ & - & \\
\hline Never married & $3(0.7 \%)$ & - & \\
\hline Duration of education, years & $9.3 \pm 2.7$ & $10.9 \pm 3.2$ & $<0.001$ \\
\hline Living location & & & 0.83 \\
\hline Urban & $305(75.3 \%)$ & $751(76.2 \%)$ & \\
\hline Rural & $100(24.7 \%)$ & $234(23.8 \%)$ & \\
\hline Living accommodation & & & $<0.001$ \\
\hline Alone in own home & $199(49.1 \%)$ & $5(0.5 \%)$ & \\
\hline With spouse in own home & $63(15.6 \%)$ & $962(97.8 \%)$ & \\
\hline With other in own home & $101(24.9 \%)^{\mathrm{c}}$ & $4(0.4 \%)$ & \\
\hline Not in own home & $42(10.4 \%)$ & $13(1.3 \%)$ & \\
\hline Number of comorbidities & $1.5 \pm 1.2$ & $1.4 \pm 1.2$ & 0.30 \\
\hline Time since AD diagnosis, years & $2.0(2.1)$ & $2.3(2.3)$ & $<0.001$ \\
\hline Experienced a fall in the last 3 months & & & $<0.001$ \\
\hline Yes & $76(18.9 \%)$ & $102(10.4 \%)$ & \\
\hline No & $327(81.1 \%)$ & $883(89.6 \%)$ & \\
\hline \multicolumn{4}{|l|}{ Caregivers } \\
\hline Age, years & $54.1 \pm 8.1$ & $73.4 \pm 8.0$ & $<0.001$ \\
\hline Female gender & $302(74.6 \%)$ & $579(58.8 \%)$ & $<0.001$ \\
\hline Marital status & & & $<0.001$ \\
\hline Married & $262(64.7 \%)$ & $984(99.9 \%)$ & \\
\hline Widowed & $24(5.9 \%)$ & 0 & \\
\hline Divorced & $59(14.6 \%)$ & $1(0.1 \%)$ & \\
\hline Never married & $60(14.8 \%)$ & 0 & \\
\hline Lives with patient & & & $<0.001$ \\
\hline Yes & $106(26.2 \%)$ & $977(99.2 \%)$ & \\
\hline No & $299(73.8 \%)$ & $8(0.8 \%)$ & \\
\hline Sole caregiver & & & $<0.001$ \\
\hline Yes & $110(27.2 \%)$ & $709(72.0 \%)$ & \\
\hline No & $295(72.8 \%)$ & $276(28.0 \%)$ & \\
\hline Employment status & & & $<0.001$ \\
\hline Not working & $169(41.7 \%)$ & $901(91.5 \%)$ & \\
\hline Working & $236(58.3 \%)$ & $84(8.5 \%)$ & \\
\hline Number of medical conditions & $0.5 \pm 0.8$ & $1.3 \pm 1.2$ & $<0.001$ \\
\hline
\end{tabular}

Data presented as means \pm SD or numbers with percentages in parentheses. Percentages are based on the number of patients with data. $p$ values in bold are significant.

${ }^{a}$ Cochran-Mantel-Haenszel test (categorical variables) or GLM type III sum of squares for relationship (continuous variables). Models stratified by caregiver-patient relationship, country and MMSE severity group.

${ }^{\mathrm{b}}$ Marital status is not reported for the group of patients who were cared for by their spouse because by definition their marital status has to be married. Relationship status was collected in the RUD questionnaire, which does not specify how people who are partners/cohabiting with the patient should respond and they may be included in the spousal group with husbands and wives.

${ }^{c}$ For these patients, some of the 'other' persons they live with will be the adult-child caregiver. 
Reed et al.: Caregiver Burden in Alzheimer's Disease: Differential Associations in Adult-Child and Spousal Caregivers in the GERAS Observational Study

Table 2. Clinical factors for patients and caregivers by caregiver relationship to the patient

\begin{tabular}{|c|c|c|c|c|}
\hline & \multirow{2}{*}{$\begin{array}{l}\text { Adult-child caregiver } \\
(n=405)\end{array}$} & \multirow{2}{*}{$\begin{array}{l}\text { Spousal caregiver } \\
(n=985)\end{array}$} & \multicolumn{2}{|c|}{ Group difference $^{a}$} \\
\hline & & & difference & $\mathrm{p}$ value \\
\hline \multicolumn{5}{|l|}{ Patient factors } \\
\hline \multicolumn{5}{|l|}{ ADCS-ADL } \\
\hline Total ADL score & $46.5 \pm 19.6$ & $46.4 \pm 19.5$ & 1.48 & 0.11 \\
\hline Basic ADL score & $16.9 \pm 5.6$ & $17.4 \pm 5.1$ & -0.27 & 0.29 \\
\hline Instrumental ADL score & $29.5 \pm 14.8$ & $28.9 \pm 15.4$ & 1.70 & 0.018 \\
\hline \multicolumn{5}{|l|}{ ADCS-ADL subdomain } \\
\hline Household activities & $16.8 \pm 8.9$ & $14.9 \pm 8.8$ & 2.48 & $<0.001$ \\
\hline Basic activities & $15.0 \pm 4.6$ & $15.9 \pm 4.2$ & -0.72 & $<0.001$ \\
\hline Communication & $8.3 \pm 4.9$ & $9.3 \pm 5.6$ & -0.52 & 0.06 \\
\hline Outdoor activities & $5.9 \pm 3.3$ & $6.7 \pm 8.3$ & -0.70 & 0.10 \\
\hline MMSE severity ${ }^{\mathrm{b}}$ & $17.0 \pm 6.0$ & $17.6 \pm 6.5$ & -0.59 & 0.12 \\
\hline ADAS-cog $(1-11)^{c}$ & $23.5 \pm 8.1$ & $22.3 \pm 7.9$ & 0.64 & 0.20 \\
\hline NPI total score & $17.5 \pm 17.4$ & $14.0 \pm 14.3$ & 2.91 & $<0.001$ \\
\hline \multicolumn{5}{|l|}{ NPI subdomain } \\
\hline Psychosis & $3.1 \pm 5.6$ & $2.2 \pm 4.3$ & 0.82 & 0.003 \\
\hline Affective & $3.5 \pm 4.6$ & $2.7 \pm 3.8$ & 0.75 & 0.002 \\
\hline Apathy & $5.5 \pm 5.5$ & $4.5 \pm 5.0$ & 0.86 & 0.004 \\
\hline Hyperactivity & $5.3 \pm 7.5$ & $4.6 \pm 6.7$ & 0.49 & 0.22 \\
\hline \multicolumn{5}{|l|}{ EQ-5D (by proxy) } \\
\hline Index score & $0.56 \pm 0.32$ & $0.65 \pm 0.28$ & -0.08 & $<0.001$ \\
\hline VAS & $60.1 \pm 21.1$ & $64.2 \pm 20.0$ & -3.5 & 0.003 \\
\hline \multicolumn{5}{|l|}{ Caregiver factors } \\
\hline ZBI total score & $31.8 \pm 15.2$ & $28.1 \pm 15.1$ & 2.98 & $<0.001$ \\
\hline NPI distress score & $9.9 \pm 9.2$ & $8.5 \pm 8.0$ & 1.15 & 0.016 \\
\hline \multicolumn{5}{|l|}{ Time spent caring, h/month } \\
\hline Basic ADL time & $35.3 \pm 77.4$ & $44.4 \pm 78.0$ & -10.67 & 0.012 \\
\hline Instrumental ADL time & $59.1 \pm 77.8$ & $87.9 \pm 88.0$ & -30.16 & $<0.001$ \\
\hline Supervision time & $70.7 \pm 140.5$ & $116.2 \pm 179.5$ & -53.12 & $<0.001$ \\
\hline Overall caregiver time & $138.1 \pm 171.3$ & $211.4 \pm 195.3$ & -82.3 & $<0.001$ \\
\hline \multicolumn{5}{|l|}{ EQ-5D } \\
\hline Index score & $0.85 \pm 0.20$ & $0.79 \pm 0.22$ & 0.07 & $<0.001$ \\
\hline VAS & $78.8 \pm 17.4$ & $73.3 \pm 17.1$ & 5.94 & $<0.001$ \\
\hline
\end{tabular}

Data are presented as means \pm SD. $p$ values in bold are significant. ADAS-cog $=$ Cognitive subscale of the Alzheimer's Disease Assessment Scale; VAS = visual analog scale. Reduced functioning is indicated by lower scores for MMSE and ADCS-ADL and higher scores for ADAS-cog; increased impairment is indicated by higher scores for NPI; reduced quality of life is indicated by lower scores for EQ-5D; greater caregiver burden is indicated by higher ZBI scores.

${ }^{a}$ Least square mean differences and p values from the type III sum of squares of the GLM, which included the factors caregiver-patient relationship, MMSE severity and country.

${ }^{\mathrm{b}}$ Model includes only the factors caregiver-patient relationship and country.

${ }^{\mathrm{c}}$ For mild and moderate MMSE severity groups only.

shorter duration of disease (table 1) and better functional abilities in instrumental ADL and the subdomain of household activities, they had worse scores in the ADL basic activities subdomain, more behavioral problems (NPI total score; NPI subdomains of psychosis, affective and apathy) and a lower HRQoL (EQ-5D index and visual analog scale scores) than the patients with spousal caregivers.

Adult-child caregivers had a greater caregiver burden than the spousal caregivers (unadjusted mean ZBI total score: 31.8 vs. 28.1, respectively; $\mathrm{p}<0.001$; table 2). The adult-child 
Reed et al.: Caregiver Burden in Alzheimer's Disease: Differential Associations in Adult-Child and Spousal Caregivers in the GERAS Observational Study

Table 3. Factors associated with ZBI total score by caregiver relationship to patient: results from final multivariate GLMs

\begin{tabular}{|c|c|c|c|c|c|c|}
\hline & \multicolumn{3}{|c|}{$\begin{array}{l}\text { Adult-child caregiver }(\mathrm{n}=405) \\
\mathrm{R}_{\mathrm{DEV}}^{2}=0.36\end{array}$} & \multicolumn{3}{|c|}{$\begin{array}{l}\text { Spousal caregiver }(n=985) \\
\mathrm{R}^{2} \mathrm{DEV}=0.43\end{array}$} \\
\hline & estimate & $95 \%$ CL & $\mathrm{p}$ value & estimate & $95 \% \mathrm{CL}$ & $p$ value \\
\hline Intercept & 38.30 & $33.22 ; 43.39$ & $<0.001$ & 45.68 & $36.83 ; 54.52$ & $<0.001$ \\
\hline \multicolumn{7}{|l|}{ Patient factors } \\
\hline \multicolumn{7}{|l|}{ MMSE (ref.: moderately severe/severe AD) ${ }^{\mathrm{a}}$} \\
\hline Mild AD & 2.06 & $-1.51 ; 5.62$ & 0.26 & 1.97 & $-0.39 ; 4.32$ & 0.10 \\
\hline Moderate AD & 2.65 & $-0.66 ; 5.96$ & 0.12 & 1.72 & $-0.38 ; 3.82$ & 0.11 \\
\hline ADCS-ADL total score & -0.15 & $-0.23 ;-0.07$ & $<0.001$ & -0.28 & $-0.33 ;-0.23$ & $<0.001$ \\
\hline Years of education & - & - & - & 0.37 & $0.13 ; 0.60$ & 0.002 \\
\hline Living in a rural location (ref.: urban) & -3.08 & $-5.95 ;-0.21$ & 0.035 & - & - & - \\
\hline No falls in the past 3 months (ref.: at least one fall) & -4.21 & $-7.41 ;-1.01$ & 0.010 & - & - & - \\
\hline \multicolumn{7}{|l|}{ Caregiver factors } \\
\hline Caregiver female (ref.: male) & - & - & - & 2.79 & $1.25 ; 4.33$ & $<0.001$ \\
\hline Caregiver age & - & - & - & -0.25 & $-0.34 ;-0.15$ & $<0.001$ \\
\hline \multicolumn{7}{|l|}{ Caregiver does not live with patient } \\
\hline (ref.: lives with patient) & -3.27 & $-6.29 ;-0.26$ & 0.034 & - & - & - \\
\hline NPI distress score & 0.74 & $0.60 ; 0.89$ & $<0.001$ & 0.75 & $0.65 ; 0.85$ & $<0.001$ \\
\hline \multicolumn{7}{|l|}{ Country (ref.: UK) } \\
\hline Germany & -2.86 & $-5.93 ; 0.20$ & 0.07 & -2.97 & $-4.68 ;-1.26$ & 0.001 \\
\hline France & -2.90 & $-5.87 ; 0.06$ & 0.06 & 4.32 & $2.41 ; 6.22$ & $<0.001$ \\
\hline
\end{tabular}

Where the cells are blank, the variable was not entered into the final model for that group of caregivers ( $p>0.05$ in univariate analysis). A negative estimate indicates a lower caregiver burden compared with the reference category (for categorical variables) or as the value increased (for continuous variables). $\mathrm{p}$ values in bold are significant. $\mathrm{CL}=$ Confidence limit.

${ }^{a}$ MMSE and country were forced into the final models.

caregivers spent less time on all aspects of caregiving (basic ADL, instrumental ADL, and supervision) and had a better HRQoL compared with the spousal caregivers (table 2).

The results of the final GLMs (table 3) indicated that a lower caregiver burden (ZBI total score) was independently associated with better patient functional ability (higher ADCS-ADL total score) in both groups of caregivers. Disease severity (MMSE) was not independently associated with caregiver burden once other variables were taken into account. Also, a higher caregiver NPI distress score was independently associated with increased caregiver burden for both adult-child and spousal caregivers. For adult-child caregivers, a higher burden was associated with living with the patient, patient living in an urban location, and whether the patient had experienced a fall in the past 3 months. For spousal caregivers, a higher burden was associated with the caregiver being female, being younger, and when the patient had spent more years in education.

\section{Sensitivity Analyses}

When the ADCS-ADL total score was excluded from the final model, AIC statistics showed a slightly poorer model fit. Without ADCS-ADL total score in the spousal model, the MMSE severity group was significant, and caregiver time for basic ADL and instrumental ADL and disease duration were included in the model, whereas in the adult-child caregiver model, the MMSE severity group remained nonsignificant, and no additional factors entered the model. 
Reed et al.: Caregiver Burden in Alzheimer's Disease: Differential Associations in Adult-Child and Spousal Caregivers in the GERAS Observational Study

Replacing the ADCS-ADL total score with the instrumental and basic ADL subdomains showed that the association of functionality with burden was predominately associated with the instrumental subdomain for both of the caregiver-patient relationship cohorts.

Replacing the ADCS-ADL total score with the four ADL subdomains (basic activities, household activities, outdoor activities, and communication) improved the model fit (based on the AIC statistic) for both caregiver cohorts. In the spousal model, household activities and communication were statistically significant in the model, and higher scores were associated with a lower burden. In the adult-child model, only the communication subdomain was statistically significant; an increase in the scores for ADL communication was associated with a lower burden.

Inclusion of caregiver depression as a specific condition improved the model fit (based on the AIC statistic) for both the spousal and adult-child models. Higher burden scores were associated with the caregiver reporting depression.

\section{Discussion}

Our results show that two thirds of the patients with AD in the GERAS study cohort were being cared for by their spouse at the baseline assessment, and that adult-child caregivers appeared to experience a higher burden than spousal caregivers despite spending less time caregiving. Regression analysis showed that patient functioning and caregiver distress due to patient behavior were both associated with caregiver burden irrespective of the caregiverpatient relationship. Disease severity (MMSE) was not an independent determinant of caregiver burden due to the strong association between patient functional ability and disease severity. Additional patient and caregiver characteristics were differentially associated with the burden experienced by adult-child and spousal caregivers. These findings imply that the caregiver's relationship to the patient should be considered when implementing interventions for reducing caregiver burden and improving the care of community-dwelling patients with AD.

Although the GERAS study baseline results are generally consistent with those of some previous studies (e.g. Conde-Sala et al. [13]), our study has a particular strength in that it was conducted in a large cohort of community-dwelling patients, with a wide distribution of AD severity (including more severe AD), and with their family caregivers in multiple countries. Other studies tended to be conducted in individual countries, in much smaller samples, and to focus on patients with milder AD.

Consistent with data from Spain [13] and other studies [15], we found that the caregiver burden was greater in adult-child caregivers than in spousal caregivers. Subjective reasons for this have been attributed to spouses living with incremental increases in disease severity and gradually adjusting to this way of living, whereas the adult-child caregivers may experience considerable disruption to their usual lifestyle and may have to perform other family duties in addition to caring for their parent with AD [13]. In contrast, some researchers have found that spouses of patients with dementia experience a greater burden than adult-child caregivers [20, 21, 44]. For example, in Korea, spousal caregivers reported a higher burden than either adult-child caregivers or daughters-in-law, with the latter group forming the largest group of caregivers for older family members in that country [44]. Additionally, data from a population-based survey in Latin America, India, and China showed that children/children-in-law had similar ZBI scores to spousal caregivers, whereas other types of caregivers had a lower level of burden [24]. It is likely that cultural traditions in caring for frail older parents are an important factor influencing the differential findings from these studies. 
In the GERAS study, the two factors independently associated with caregiver burden in both adult-child and spousal caregivers were patient functional abilities (ADCS-ADL total score) and caregiver distress due to the patient's behavioral symptoms (NPI distress score). Better patient functioning (ADCS-ADL total score) was associated with a lower burden in both types of caregiver, although the estimates in table 3 indicate that the impact was greater on spousal caregivers, almost all of whom lived with the patient. Our results are consistent with those of Conde-Sala et al. [13], who found that greater functional ability (measured using the Disability Assessment for Dementia scale) was negatively correlated with caregiver burden for both adult-child and spousal caregivers. Both that study and the current analysis found no difference in functional ability between patients cared for by adult-child versus spousal caregivers in the univariate analysis. Our sensitivity analysis showed that instrumental ADL but not basic ADL was significantly associated with burden for both types of caregiver. Considering the four subdomains of the ADL, adult-child and spousal caregivers had a significantly higher burden with poorer scores in communication, whereas only spousal caregivers had a higher burden associated with poorer scores on household activities. Thus, spousal caregivers were impacted more by a patient's loss of ability to perform household chores, which would be consistent with most spouses being the sole caregiver and living with the patient and, therefore, having to undertake the household tasks when the patient was unable to perform them.

In previous studies, impaired instrumental ADL [20] and ADL abilities (measured using the Disability Assessment for Dementia scale) [45] were associated with caregiver burden. However, functional ability (ADCS-ADL score) was only weakly correlated with caregiver burden in an analysis of the CATIE-AD study [22]. One reason for the different findings between studies may be that the concept of ADL is closely related to MMSE severity and caregiver time, such that these variables may have a similar influence on caregiver burden. The results of the model used by Bergvall et al. [45] implied that part of the association between ADL abilities and caregiver burden was mediated by informal care hours. Our sensitivity analyses confirm the relationship between ADL, MMSE and caregiver time; however, the results indicate that ADL is a better explanatory variable for caregiver burden than either of the other two variables.

Research has consistently shown that AD patient behavioral problems (neuropsychiatric symptoms) have a substantial impact on the caregiver and are associated with increased caregiver burden $[13,14,20,29,45-47]$. Some of these studies have reported that caregiver burden/distress is associated with specific behavioral disturbances, but the findings vary between studies. In the GERAS study, adult-child caregivers reported significantly greater patient impairment in the NPI subdomains of psychosis, affective and apathy compared with spousal caregivers. In the regression model, greater caregiver distress due to the patient's behavioral symptoms (NPI distress score) was associated with greater caregiver burden in both caregiver-patient relationships. These findings suggest that treatments for reducing patient behavioral symptoms are an important therapeutic option and may not only alleviate patient suffering, but may also lead to a reduction in the perceived burden of caregivers.

Although the number of medical conditions in caregivers was not found to be significant in the burden analysis for either spousal or adult-child caregivers, when we explored caregiver depression as a specific condition it was found to be associated with burden for both types of caregiver. In a previous study, depressive symptoms were related to the burden of adult-child caregivers but not of spousal caregivers [13].

Our analyses identified differential factors associated with adult-child and spousal caregiver burden. Caregiver gender and age were associated with spousal caregiver burden: wife caregivers and younger spouse caregivers reported a greater burden. The gender difference in spousal caregiver burden is in agreement with previous studies $[13,48]$, and may be 
Reed et al.: Caregiver Burden in Alzheimer's Disease: Differential Associations in Adult-Child and Spousal Caregivers in the GERAS Observational Study

mediated by different coping strategies [48]. Male spousal caregivers may also receive more support from other family members or professional services [49]. Other researchers have found that younger caregivers can experience a higher level of burden [20]. Spousal caregiver burden was also greater when caring for patients with more years of education, which has not been reported previously, although the estimate for this variable is quite small for each change in burden score. Considered together with the findings on caregiver gender and age, we could hypothesize that caregivers may have expectations of patients with more years of education to fulfill specific aspects of daily living (e.g. financial or emotional aspects), and these caregivers are now struggling with their change in role and their ability to provide support for their spouse.

Our results showed that adult-child caregivers who live with the patient $(26.2 \%$ of the adult-child caregiver group) had a higher burden than those who do not live with the patient, supporting the findings of Conde-Sala et al. [13]. One possible reason for this difference may be that caregivers who live with a parent with $\mathrm{AD}$ have greater difficulties in managing the situation [13], especially if they have family and/or work responsibilities, and may have less personal time and experience more social isolation from friends. Research into the impact of marital status in adult-child caregivers on burden may be useful to further assess this finding. Another interesting finding is the association between greater adult-child caregiver burden and whether patients had experienced a fall in the previous 3 months. Falls are common in elderly people with dementia and increase patient dependence on their caregiver for basic ADL [50]. More falls can also be considered a measure of worse patient physical health and can result in reduced mobility, which would be expected to lead to increased caregiver time spent caring for the patient, thereby potentially increasing the caregiver burden. In a prospective cohort study in Italy, caregiver burden was associated with the AD patient's risk of falling, and there was a higher risk of falls among patients whose caregivers were nonspouse and non-first-degree relatives [51]. These researchers speculated that caregiver emotional and psychological distress might contribute to patient falls. Although further research is needed to elucidate the causal relationship, both findings could imply that interventions aimed at reducing caregiver burden, especially among adult-child caregivers, should include training and strategies for preventing patient falls.

The analyses presented in the current article focus on the subjective emotional aspects of caregiver burden assessed using the ZBI. Other aspects of caregiver burden, such as caregiver health (physical and mental) [12], time spent caring or the costs of informal care [27], may be affected by other factors than those reported here.

Several limitations of the study must be considered when interpreting the results. First, this was a selected sample of community-dwelling AD patients and may not represent the full spectrum of caregiver burden, although patients with mild, moderate and moderately severe/ severe AD were included. Second, although we have identified several patient and caregiver factors associated with subjective caregiver burden, we cannot assume causality and it remains unclear whether these factors are a cause or consequence of caregiver burden. Third, there may be some rating bias in the caregiver assessment of patient behavioral symptoms (NPI) and HRQoL (EQ-5D) as it has been shown that the health and well-being of caregivers can influence their evaluation of the patient; caregiver burden and depression have been associated with more negative assessments of these patient factors [52]. Moreover, the worse patient behavioral symptoms (higher NPI total score) in the adult-child caregiver cohort indicate potential selection bias, such that patients living with spouses with very severe behavioral symptoms may have already been institutionalized. Fourth, although HRQoL is a potentially important variable affecting caregiver burden, it was not well measured by the EQ-5D. We did not include EQ-5D scores in the GLMs examining factors associated with the ZBI total score because patient HRQoL is a proxy measure completed by the caregiver and so 
Reed et al.: Caregiver Burden in Alzheimer's Disease: Differential Associations in Adult-Child and Spousal Caregivers in the GERAS Observational Study

may be too complex to associate with the caregiver feeling of burden. Also, the baseline caregiver EQ-5D score has a skewed distribution, which will make any association with caregiver burden difficult to interpret. Employing a disease-specific HRQoL scale may have been more useful. In their analysis of the differences between adult-child and spousal caregivers in the perceived quality of life of patients using the Quality of Life in AD scale, Conde-Sala et al. [53] found that adult-child caregivers had a more negative perception of patient quality of life compared with spousal caregivers, and this was associated with greater caregiver burden and higher levels of depression in the patient.

Our study considered a broad range of potential factors that may be associated with caregiver burden. Our baseline analyses identified differential factors associated with burden that could potentially be prevented (e.g. falls) or modified (e.g. living location) to help reduce caregiver burden. As GERAS is a longitudinal study, future analysis of changes in burden and factors associated with these changes will be useful in assessing the impact of disease progression on caregiver burden.

In conclusion, our results showed differences between the burden perceived by adultchild and spousal caregivers when caring for their relative with AD. Our data, from a large study involving patients and caregivers from three European countries, are consistent with those of Conde-Sala et al. [13], suggesting that the previous findings in caregivers of patients with milder AD [13] can be extended to caregivers of patients with more severe AD. Several patient and caregiver characteristics and clinical factors associated with caregiver burden differed between adult-child and spousal caregivers, although better patient functioning (particularly instrumental ADL) and less caregiver distress due to patient's behavioral problems were associated with a lower burden for both types of caregiver. Our findings suggest that the needs of adult-child and spousal caregivers are different and imply that interventions that are aimed at reducing the individual caregiver burden and improving patient care should be tailored to support each caregiver depending on the caregiver-patient relationship.

\section{Acknowledgements}

This study was supported by Eli Lilly and Company. The authors would like to acknowledge Dr. Deirdre Elmhirst and Dr. Claire Lavin (Rx Communications, Mold, UK) for medical writing assistance with the preparation of this article, funded by Eli Lilly and Company.

\section{Disclosure Statement}

Mark Belger, Grazia Dell'Agnello, and Catherine Reed are all employees of Eli Lilly and Company Limited. Josep M. Argimon, Giuseppe Bruno, Richard Dodel, Bruno Vellas, and Anders Wimo have received financial compensation from Eli Lilly for participation on the GERAS Advisory Board. Roy W. Jones and Josep Maria Haro are consultants for Eli Lilly.

\section{References}

1 Ballard C, Gauthier S, Corbett A, Brayne C, Aarsland D, Jones E: Alzheimer's disease. Lancet 2011;377:10191031.

2 Mayeux R, Stern Y: Epidemiology of Alzheimer disease. Cold Spring Harb Perspect Med 2012;2:a006239.

-3 Prince M, Bryce R, Albanese E, Wimo A, Ribeiro W, Ferri CP: The global prevalence of dementia: a systematic review and metaanalysis. Alzheimers Dement 2013;9:63-75. 
Reed et al.: Caregiver Burden in Alzheimer's Disease: Differential Associations in Adult-Child and Spousal Caregivers in the GERAS Observational Study

4 Wimo A, Reed CC, Dodel R, Belger M, Jones RW, Happich M, Argimon JM, Bruno G, Novick D, Vellas B, Haro JM: The GERAS study: a prospective observational study of costs and resource use in community dwellers with Alzheimer's disease in three European countries - study design and baseline findings. J Alzheimers Dis 2013; 36:385-399.

5 Rapp T, Andrieu S, Molinier L, Grand A, Cantet C, Mullins CD, Vellas B: Exploring the relationship between Alzheimer's disease severity and longitudinal costs. Value Health 2012;15:412-419.

-6 Beinart N, Weinman J, Wade D, Brady R: Caregiver burden and psychoeducational interventions in Alzheimer's disease: a review. Dement Geriatr Cogn Disord Extra 2012;2:638-648.

7 Burns A: The burden of Alzheimer's disease. Int J Neuropsychopharmacol 2000;3:31-38.

8 Balardy L, Voisin T, Cantet C, Vellas B, REAL.FR Group: Predictive factors of emergency hospitalisation in Alzheimer's patients: results of one-year follow-up in the REAL.FR cohort. J Nutr Health Aging 2005;9:112-116.

$\triangleright 9$ Brodaty H, McGilchrist C, Harris L, Peters KE: Time until institutionalization and death in patients with dementia. Role of caregiver training and risk factors. Arch Neurol 1993;50:643-650.

10 Brodaty H, Donkin M: Family caregivers of people with dementia. Dialogues Clin Neurosci 2009;11:217-228.

11 Schulz R, Beach SR: Caregiving as a risk factor for mortality: the Caregiver Health Effects Study. JAMA 1999; 282:2215-2219.

12 Schulz R, Sherwood PR: Physical and mental health effects of family caregiving. Am J Nurs 2008;108(9 suppl):23-27.

13 Conde-Sala JL, Garre-Olmo J, Turró-Garriga O, Vilalta-Franch J, López-Pousa S: Differential features of burden between spouse and adult-child caregivers of patients with Alzheimer's disease: an exploratory comparative design. Int J Nurs Stud 2010;47:1262-1273.

14 Mohamed S, Rosenheck R, Lyketsos CG, Schneider LS: Caregiver burden in Alzheimer disease: cross-sectional and longitudinal patient correlates. Am J Geriatr Psychiatry 2010;18:917-927.

15 Hayslip B, Han G, Anderson CL: Predictors of Alzheimer's disease caregiving depression and burden: what noncaregiving adults can learn from active caregivers. Educ Gerontol 2008;34:945-969.

16 Molyneux GJ, McCarthy GM, McEniff S, Cryan M, Conroy RM: Prevalence and predictors of carer burden and depression in carers of patients referred to an old age psychiatric service. Int Psychogeriatr 2008;20:11931202.

17 Andren S, Elmstahl S: Relationships between income, subjective health and caregiver burden in caregivers of people with dementia in group living care: a cross-sectional community-based study. Int J Nurs Stud 2007;44: 435-446.

18 Coen RF, O’Boyle CA, Coakley D, Lawlor BA: Individual quality of life factors distinguishing low-burden and high-burden caregivers of dementia patients. Dement Geriatr Cogn Disord 2002;13:164-170.

19 Kim H, Chang M, Rose K, Kim S: Predictors of caregiver burden in caregivers of individuals with dementia. J Adv Nurs 2011;68:846-855.

20 Germain S, Adam S, Olivier C, Cash H, Ousset PJ, Andrieu S, Vellas B, Meulemans T, Reynish E, Salmon E, the ICTUS-EADC Network: Does cognitive impairment influence burden in caregivers of patients with Alzheimer's disease? J Alzheimers Dis 2009;17:105-114.

21 Andrén S, Elmståhl S: The relationship between caregiver burden, caregivers' perceived health and their sense of coherence in caring for elders with dementia. J Clin Nurs 2008;17:790-799.

-22 Serrano-Aguilar PG, Lopez-Bastida J, Yanes-Lopez V: Impact on health-related quality of life and perceived burden of informal caregivers of individuals with Alzheimer's disease. Neuroepidemiology 2006;27:136-142.

-23 Rinaldi P, Spazzafumo L, Mastriforti R, Mattioli P, Marvardi M, Polidori MC, Cherubini A, Abate G, Bartorelli L, Bonaiuto S, Capurso A, Cucinotta D, Gallucci M, Giordano M, Martorelli M, Masaraki G, Nieddu A, Pettenati C, Putzu P, Tammaro VA, Tomassini PF, Vergani C, Senin U, Mecocci P, Study Group on Brain Aging of the Italian Society of Gerontology and Geriatrics: Predictors of high level of burden and distress in caregivers of demented patients: results of an Italian multicentre study. Int J Geriatr Psychiatry 2005;20:168-174.

-24 Prince M, Brodaty H, Uwakwe R, Acosta D, Ferri CP, Guerra M, Huang Y, Jacob KS, Llobre Rodriguez JJ, Salas A, Sosa AL, Williams JD, Jotheeswaran AT, Liu Z: Strain and its correlates among carers of people with dementia in low-income and middle-income countries. A 10/66 Dementia Research Group population-based survey. Int J Geriatr Psychiatry 2012;27:670-682.

25 Ankri J, Andrieu S, Beaufils B, Grand A, Henrard JC: Beyond the global score of the Zarit Burden Interview: useful dimensions for clinicians. Int J Geriatr Psychiatry 2005;20:254-260.

-26 Ferrario SR, Vitaliano P, Zotti AM, Galante E, Fornara R: Alzheimer's disease: usefulness of the Family Strain Questionnaire and the Screen for Caregiver Burden in the study of caregiving-related problems. Int J Geriatr Psychaitry 2003;18:1110-1114.

-27 Wolfs CA, Kessels A, Severens JL, Brouwer W, de Vugt ME, Verhey FR, Dirksen CD: Predictive factors for the objective burden of informal care in people with dementia: a systematic review. Alzheimer Dis Assoc Disord 2012;26:197-204.

28 Campbell P, Wright J, Oyebode J, Job D, Crome P, Bentham P, Jones L, Lendon C: Determinants of burden in those who care for someone with dementia. Int J Geriatr Psychiatry 2008;23:1078-1085.

29 Allegri RF, Sarasola D, Serrano CM, Arizaga RL, Butman J, Lon L: Neuropsychiatric symptoms as a predictor of caregiver burden in Alzheimer's disease. Neuropsychiatr Dis Treat 2006;2:105-110.

-30 Zarit SH, Reever KE, Bach-Peterson J: Relatives of the impaired elderly: correlates of feelings of burden. Gerontologist 1980;20:649-655. 
Reed et al:: Caregiver Burden in Alzheimer's Disease: Differential Associations in Adult-Child and Spousal Caregivers in the GERAS Observational Study

31 Marwit SJ, Meuser TM: Development and initial validation of an inventory to assess grief in caregivers of persons with Alzheimer's disease. Gerontologist 2002;42:751-765.

32 Haro JM, Kahle-Wrobleski K, Bruno G, Belger M, Grazia Dell'Agnello G, Dodel R, Jones RW, Reed CC, Vellas B, Wimo A, Argimon JM: Analysis of burden in caregivers of people with Alzheimer's disease using self-report and supervision hours. J Nutr Health Aging 2014, Epub ahead of print.

-33 McKhann G, Drachman D, Folstein M, Katzman R, Price D, Stadlan EM: Clinical diagnosis of Alzheimer's disease: report of the NINCDS-ADRDA Work Group under the auspices of Department of Health and Human Services Task Force on Alzheimer's Disease. Neurology 1984;34:939-944.

-34 Folstein MF, Folstein SE, McHugh PR: 'Mini-mental state'. A practical method for grading the cognitive state of patients for the clinician. J Psychiatr Res 1975;12:189-198.

35 National Institute of Health and Clinical Excellence: NICE technology appraisal guideline 217. Donepezil, galantamine, rivastigmine and memantine for the treatment of Alzheimer's disease. http://www.nice.org.uk/ guidance/TA217 (accessed September 2013).

-36 Rosen WG, Mohs RC, Davis KL: A new rating scale for Alzheimer's disease. Am J Psychiatry 1984;141:13561364.

37 Galasko D, Schmitt F, Thomas R, Jin S, Bennett D: Alzheimer's Disease Cooperative Study: detailed assessment of activities of daily living in moderate to severe Alzheimer's disease. J Int Neuropsychol Soc 2005;11:446453.

-38 Cummings JL: The Neuropsychiatric Inventory: assessing psychopathology in dementia patients. Neurology 1997;48(5 suppl 6):S10-S16.

39 Kaufer DI, Cummings JL, Christine D, Bray T, Castellon S, Masterman D, MacMillan A, Ketchel P, DeKosky ST: Assessing the impact of neuropsychiatric symptoms in Alzheimer's disease: the Neuropsychiatric Inventory Caregiver Distress Scale. J Am Geriatr Soc 1998;46:210-215.

40 The EuroQol Group: Euro-QoL - a new facility for the measurement of health-related quality of life. Health Policy 1990;16:199-208.

41 Wimo A, Wetterholm AL, Mastey V, Winblad B: Evaluation of the resource utilization and caregiver time in anti-dementia drug trials - a quantitative battery; in Wimo A, Jonsson B, Karlsson G, Winblad B (eds): The Health Economics of Dementia. London, Wiley, 1998, pp 465-499.

42 Fisher GG, Franks MM, Plassman BL, Brown SL, Potter GG, Llewellyn D, Rogers MA, Langa KM: Caring for individuals with dementia and cognitive impairment, not dementia: findings from the aging, demographics and memory study. J Am Geriatr Soc 2011;59:488-494.

43 Cooper C, Katona C, Orrell M, Livingston G: Coping strategies, anxiety and depression in caregivers of people with Alzheimer's disease. Int J Geriatr Psychiatry 2008;23:929-936.

44 Hong GR, Kim H: Family caregiver burden by relationship to care recipient with dementia in Korea. Geriatr Nurs 2008;29:267-274.

45 Bergvall N, Brinck P, Eek D, Gustavsson A, Wimo A, Winblad B, Jönsson L: Relative importance of patient disease indicators on informal care and caregiver burden in Alzheimer's disease. Int Psychogeriatr 2011;23: 73-85.

46 Kochhann R, Borba E, Cerveira MO, Onyszko D, de Jesus A, Forster L, Franciscatto L, Godinho C, Camozzato AL, Chaves MLF: Neuropsychiatric symptoms as the main determinant of caregiver burden in Alzheimer's disease. Dement Neuropsychol 2011;5:203-208.

47 Cheng ST, Lam LCW, Kwok T: Neuropsychiatric symptom clusters of Alzheimer disease in Hong Kong Chinese: correlates with caregiver burden and depression. Am J Geriatr Psychiatry 2013;21:1029-1037.

48 Poysti MM, Laakkonen ML, Strandberg T, Savikko N, Tilvis RS, Eloniemi-Silkava U, Pitkala KH: Gender differences in dementia spousal caregiving. Int J Alzheimers Dis 2012;2012:162960.

49 Miller N, Guo S: Social support for spouse caregivers of persons with dementia. J Gerontol B Psychol Sci Soc Sci 2000;55:S163-S172.

50 Salva A, Roque M, Rojano X, Inzitari M, Andrieu S, Schiffrin EJ, Guigoz Y, Vellas B: Falls and risk factors for falls in community-dwelling adults with dementia (NutriAlz trial). Alzheimer Dis Assoc Disord 2012;26:74-80.

51 Maggio D, Ercolani S, Andreani S, Ruggiero C, Mariani E, Mangialasche F, Palmari N, Mecocci P: Emotional and psychological distress of persons involved in the care of patients with Alzheimer disease predicts falls and fracture in their care recipients. Dement Geriatr Cogn Disord 2010;30:33-38.

-52 Conde-Sala JL, Rene-Ramirez R, Turro-Garriga O, Gascon-Bayarri J, Juncadella-Puig M, Moreno-Cordon L, Vinas-Diez V, Vilalta-Franch J, Garre-Olmo J: Factors associated with the variability in caregiver assessments of the capacities of patients with Alzheimer disease. J Geriatr Psychiatry Neurol 2013;26:86-94.

53 Conde-Sala JL, Garre-Olmo J, Turró-Garriga O, Vilalta-Franch J, López-Pousa S: Quality of life of patients with Alzheimer's disease: differential perceptions between spouse and adult-child caregivers. Dement Geriatr Cogn Disord 2010:29:97-108. 